\title{
Être Chez-soi en Afrique de l'Est
}

Enjeux de territorialisation et rapport à la ville (Arusha-Tanzanie)

Mathilde de Blignières

\section{(2) OpenEdition}

Journals

Édition électronique

URL : https://journals.openedition.org/eastafrica/611

DOI : $10.4000 /$ eastafrica. 611

ISSN : 2790-1076

Éditeur

IFRA - Institut Français de Recherche en Afrique

Édition imprimée

Date de publication : 1 juin 2008

Pagination : 125-150

ISSN : 2071-7245

Référence électronique

Mathilde de Blignières, «Être Chez-soi en Afrique de l'Est », Les Cahiers d'Afrique de l'Est / The East African Review [En ligne], 39 | 2008, mis en ligne le 07 mai 2019, consulté le 09 décembre 2021. URL : http://journals.openedition.org/eastafrica/611; DOI : https://doi.org/10.4000/eastafrica.611

Ce document a été généré automatiquement le 9 décembre 2021

Les Cahiers d'Afrique de l'Est / The East African Review 


\title{
Être Chez-soi en Afrique de l'Est
}

\author{
Enjeux de territorialisation et rapport à la ville (Arusha-Tanzanie)
}

\section{Mathilde de Blignières}

\section{Introduction générale}

1 Nous faisons ici une synthèse des questions « motrices » qui rythment l'ethnographie en cours menée à partir de la ville d'Arusha et de ses alentours dans le nord de la Tanzanie sur le thème du chez-soi. Nous prenons comme axes de visée l'habiter et la vie quotidienne (ou la vie quotidienne dans laquelle se manifeste l'habiter) en interrogeant l'acteur sur son environnement dans une démarche introspective et critique. L'échange se fait en partie au sein des lieux d'habitation. C'est d'abord le rapport à l'espace que nous questionnons: quel rapport entretient l'individu au territoire et de quelle territorialité s'agit-il (intime, régionale, urbaine, villageoise, nationale...)? Puis le rapport au temps, celui de l'expérience passée, du moment présent et des imaginaires projetés. Enfin le rapport aux Autres et à la société car "c'est dans l'affirmation d'un " nous » face aux « autres » que le «moi » exprime son ancrage territorial ${ }^{1}$ ». Nous souhaitons donc inscrire notre problématique du chez soi dans une analyse tridimensionnelle société-espace-temps.

2 «Chez-soi ", «être chez-soi aujourd'hui à Arusha»: le sujet d'étude est immense, l'éventail d'interlocuteurs est (par choix) très étendu, les questions posées et les univers discutés revêtent une dimension personnelle. Quant à la situation d'intrusion du chercheur chez l'acteur, elle est souvent inhabituelle et intime. Face à ces difficultés et par souci de méthode nous avons choisi de nous raccrocher au domaine du réel en questionnant les formes concrètes d'habiter dans la Tanzanie d'aujourd'hui. Car en insistant d'emblée sur le support bâti et son organisation, nous voulons rendre compte de ce que disent les maisons d'ici ou de là-bas dans leurs configurations matérielles, leur occupation et leur décoration en complément de ce que peuvent en dire euxmêmes les habitants. L'objectif est d'aboutir à une ébauche de réflexion finale sur l'autonomie de l'individu, les possibilités de réalisation de soi et d'intégration sociale qui lui sont offertes aujourd'hui en Tanzanie. 
3 Après avoir brièvement rappelé dans quel contexte et avec quels interlocuteurs l'enquête a lieu, nous entrerons dans le vif du sujet par la maison. À partir du concept de "maison domus » qui permet de lire l'espace habitable et habité dans une même veine, nous évoquerons la nécessité d'observer le chez-soi dans son organisation pragmatique en nous arrêtant sur le décor, les activités du ménage, le voisinage et la maison dans sa dispersion spatiale.

4 Une fois l'épaisseur du quotidien domestique mise en évidence, nous aborderons le domaine plus abstrait des territoires et imaginaires construits du chez-soi en examinant comment l'individu se territorialise à partir de ses propres expériences spatiales et de quels territoires il s'agit. Par le discours et l'affect, nous évoquerons ceux de l'intimité, de l'ancrage ou de la mobilité, de l'ici et de l'ailleurs afin d'avancer dans la compréhension des identités spatiales et sociales.

5 Enfin le troisième point d'accroche de notre synthèse abordera la question des pratiques et représentations des habitants interrogés vis-à-vis de la ville d'Arusha et des espaces de vie urbains en général, la ville étant le cadre d'étude propice à l'illustration des mobilités et pratiques résidentielles translocales, transnationales. Nous verrons comment celles-là entrent en jeu dans la définition du/des chez-soi et quel est le lien, en pratique comme dans l'imaginaire, entre le monde du chez-soi et celui de la ville. Avec la diversité des points de vue sur la ville et l'observation de ses différentes pratiques par les habitants, ce sera l'occasion d'inscrire notre problématique au cœur des dynamiques urbaines contemporaines.

6 C'est donc en transposant notre objet d'étude « chez-soi » au cœur de l'espace de vie, de l'espace vécu, de l'espace social, pratiqué, imaginé et en confrontant ces différentes trajectoires entre elles que nous aimerions procéder.

\section{Le contexte / les acteurs}

7 Bien qu'il s'agisse ici d'une synthèse, nous souhaitons rappeler le contexte au sein duquel les recherches prennent place ainsi que les principaux enjeux socio-historiques qui caractérisent la region d'étude. Nous resituerons également nos choix concernant le profil et le parcours de nos interlocuteurs.

\subsection{Le potentiel attractif de la ville d'Arusha}

D'après les historiens sur la région, la ville d'Arusha se caractérise depuis ses débuts par un fort potentiel attractif. Au début du Xix siècle, le futur site était un lieu de rencontres et d'échanges entre les éleveurs maasaïs et les marchands des caravanes qui rejoignaient la côte de l'océan Indien depuis l'intérieur du continent. Le déclin de la traite contribua à ce que de nouveaux arrivants, séduits par cette région fertile et bien arrosée, s'installèrent avant que les Allemands fassent le choix d'y établir leur garnison. Reprise par les Anglais courant $\mathrm{xx}^{\mathrm{e}}$ siècle, la ville ainsi que les flans du mont Meru accueillirent tour à tour plusieurs communautés de fermiers et missionnaires (Grecs, Polonais, Anglais, Américains...), les bases d'un centre touristique voué à une importante expansion, et le centre de conférence international d'Arusha qui abrite pour l'heure le siège administratif de la Communauté est-africaine, le Tribunal Pénal International pour le Rwanda et la Cour africaine de justice. Les Anglais développèrent 
la ville dans le but d'en faire un centre d'abord régional avec le siège de leur administration, puis commercial (à destination des colons européens), ainsi qu'un modèle de ville moderne en Afrique de l'Est. Le paysage urbain qui en résulta devint très vite contrasté, à l'image d'une ville coloniale classique marquée par la ségrégation raciale avec son quartier européen, son centre-ville marchand ici contrôlé par les commerçants d'origines indiennes et sa ceinture verte séparant les quartiers résidentiels des «bazars » africains et de la zone industrielle. Bien que de forts conflits idéologiques sur l'espace accompagnassent le développement de la ville, celle-ci n'en resta pas moins caractérisée par un cosmopolitisme permanent, visible dans l'espace urbain.

9 Ce survol historique remet à jour les différentes phases du peuplement de la ville d'Arusha depuis ses débuts en tant qu'agglomération urbaine. Avant l'indépendance du pays en 1961, Arusha a toujours été une ville d'étrangers (officiers coloniaux européens, marchands asiatiques, grecs, commerçants et travailleurs Chagga, Pare, Somali et swahilis.). Aujourd'hui, en plus des migrations transnationales qui contribuent à attirer plusieurs Tanzaniens venus de régions diverses, la présence de nombreux fonctionnaires internationaux, entrepreneurs est/ouest/sud-africains, occidentaux, asiatiques continue de faire la particularité de la ville et d'asseoir sa réputation de centre d'affaires actif en Afrique de l'Est.

Arusha occupe une place centrale au cœur de nos recherches en tant qu'agglomération urbaine fédératrice des différents chez-soi observés. Néanmoins la ville de Moshi et ses alentours font également l'objet de terrains ponctuels tandis que sont constamment évoquées les autres régions du pays dans le discours de nos interlocuteurs.

\subsection{Qui sont les acteurs?}

11 En écho au cosmopolitisme de la ville d'Arusha, nous avons choisi de privilégier une approche élargie du choix de nos interlocuteurs. Nous partons de l'idée que tout individu rencontré - informé de l'enquête et désireux d'y contribuer - est acteur potentiel quel que soit son âge, son profil social, économique, ses activités, ses antécédents. L'hétérogénéité de ces rencontres est un facteur déterminant pour nos recherches car nous considérons d'abord tout habitant, qu'il soit migrant réel ou potentiel, comme producteur de formes très diverses d'habiter; d'autre part parce que nous ne prétendons pas mener d'étude sociologique au départ de profils économiques types. À partir de là, nos priorités concernent la réhabilitation de l'acteur et sa propre restitution en tant que sujet pensant dans la société moderne tanzanienne en questionnant la façon dont celui-ci se pense, se (re)présente et se revendique (est-ce en qualité passive, active ?), ainsi que la remise en circuit du contexte « maison ».

\section{Le chez-soi dans ses formes concrètes}

\subsection{Réhabiliter la structure maison}

12 Avec la globalisation économique et les nouveaux réseaux de circulation dont il est désormais question à l'échelle planétaire, la recherche en sciences sociales est affublée, si ce n'est en demande, d'un champ sémantique de plus en plus complexe. D'après le sociologue Alain Tarrius, "(...) l'urgence consiste à produire des notions, des modes 
d'observation et de description, qui rendent compte des articulations et simultanéités des mouvements généralisés redéfinissant les règles de "l'être ensemble ", dans les côtoiements des voisinages momentanés (...) et durables ${ }^{22}$ ». Il est question par exemple de ces subtilités dans les notions de "territoires circulatoires", "réseaux transnationaux", "télescopages entre l'ici et l'ailleurs", "superpositions territoriales", "nouveaux cosmopolitismes", etc. Alors que la mobilité à grande échelle est de plus en plus connotée positivement ou considérée comme un critère de modernité, nous tenons à réhabiliter la place de la maison dans l'existence des individus, laquelle maison est souvent tenue à l'écart de ces nouveaux mouvements passionnants.

Le support maison nous est ici utile à plusieurs niveaux. D'abord il rend l'habiter repérable dans l'espace pour le chercheur comme pour son interlocuteur. Il propose pour l'un de rester en phase avec le réel et pour l'autre un moyen visible d'accéder à la démarche scientifique, facilitant par là l'échange interculturel. La connaissance de la maison s'avère également indispensable à la lecture des sociabilités contemporaines (et notamment citadines). Nous supposons qu'elle abrite à différents degrés de la société des clefs de compréhension des modes de vie, rêves et aspirations, difficultés, motivations (et autres) des individus ou des groupes. Même mise en scène, la maison témoigne d'une spontanéité qui met à nu l'habitant en ce qu'elle est rythmée par le quotidien pragmatique, banal et routinier qui l'organise. Bien qu'elle soit « enracinée dans la terre ", étudier la maison ne revient pas à négliger les capacités circulatoires de l'individu ni à réduire ses horizons par une sédentarisation forcenée. Au contraire, si l'attachement au lieu fait toujours sens et celui aux origines constamment réactualisé, la maison est source de dynamisme en ce qu'elle regroupe à elle seule l'être d'ici, l'être de là-bas, l'être d'ici et de là-bas à la fois.

\subsection{La « maison-domus »}

14 La vitalité de l'objet donné prend corps à travers l'exemple de "maison-domus" réhabilité par les socio-anthropologues de l'habitat en Europe. Ces derniers ont permis de confronter dans une même analyse ce qui fait le groupe domestique (la maisonnée), son espace matériel (la maison) et ses ressources, et de fournir par là une structure sociale intermédiaire qui paraît mieux à même de rendre compte des stratégies d'acquisition, d'occupation, de transmission patrimoniale, de leur articulation avec l'individuel et le macro-social. La maison-domus est pour nous d'autant plus pertinente qu'elle s'instrumentalise et s'identifie dans l'habitation proprement dite, donc dans l'espace concret convoité précédemment. Elle peut aussi être décrite et analysée à trois niveaux : celui du capital localisé qu'elle représente (possédé ou non par l'occupant luimême), celui de l'espace habitable, fonctionnalisé, comme instrument nécessaire aux pratiques domestiques quotidiennes et festives, répétitives ou exceptionnelles, enfin celui de l'expression symbolique et identitaire dont elle est le support.

En facilitant l'analyse croisée habitat/habitant/pratiques habitantes, la maison-domus fournit un cadre d'étude propice à l'anthropologie du chez-soi, notamment en contexte résidentiel tanzanien où les stratégies collectives sont encore largement privilégiées par rapport aux formes de repli et d'individuation. 


\subsection{L'observation des pratiques habitantes}

«Pour comprendre les pratiques habitantes, pratiques de consommation et pratiques productives, dans leurs dimensions matérielles et symboliques, il faut avant tout considérer que s'opère un attachement, une identification, socialement attendue, entre le groupe domestique et son espace quotidien. Tout en produisant sa propre existence, le ménage investit une part de ses ressources et de son énergie, et contribue à produire son espace matériel non pas comme un objet détaché de lui, mais bien comme une modalité de sa propre existence ${ }^{3}$.»

Pour effectuer la synthèse de nos idées phares, nous avons d'abord rappelé la nécessité de marquer l'arrêt sur la maison en soulignant les dynamiques que celle-ci fédère audelà de son immobilité apparente. Nous poursuivons sa réhabilitation en nous plaçant ici du côté du chercheur introduit chez l'habitant et des images habitées qui lui sont données à voir. L'objectif est de rappeler l'importance de chaque champ d'observation (qu'il s'agisse du décor, de la fonctionnalité des lieux, des rapports avec le voisinage ou encore de la multi-résidence), et de leurs interactions avec les mondes extérieurs. Nous avons pour souci de resituer concrètement la place de chacun (enquêteur et enquêté) dans la situation d'exploration tout en examinant comment l'acteur habite les lieux mais aussi de quelle manière ceux-ci l'habitent.

\subsubsection{Le décor}

17 Au-delà de la structure globale bâtie, le décor se situe dans les premiers instants de l'observation du chez-soi. Rapidement accessible, il est donné à voir jusque dans ses moindres détails. Qu'il soit caractérisé par une accumulation exagérée des choses ou un franc dépouillement, il permet de comprendre les différents comportements résidentiels des ménages mais aussi l'attachement du groupe domestique à son espace quotidien. Le choix des objets, leur continuité d'une maison à l'autre, leur originalité, etc., participent à la création d'un capital culturel inscrit à son tour dans une mémoire résidentielle. Le décor est à distinguer de la configuration matérielle en ce qu'il comporte une dimension sentimentale supplémentaire. Il est analysable en tant que tel (la décoration), mais aussi pour ce qu'il exprime sur l'habitant et en complément de ce que ce dernier peut lui-même en dire.

\subsubsection{La fonctionnalité de la maison}

18 Introduit dans les lieux de l'habitation, le chercheur accède ensuite à l'observation d'un espace domestique rythmé par de nombreuses activités quotidiennes. Qu'elles soient répétitives ou exceptionnelles, celles-ci sont partie prenante du processus d'identification $\mathrm{du}$ chez-soi. Elles rendent nécessaires l'analyse voire la problématisation des espaces-temps de la consommation et de la production du ménage. Soulignons à ce titre l'absence de visibilité en sciences sociales dans le domaine des études africaines (Afrique de l'Est en particulier) des pratiques habitantes $\mathrm{du}$ quotidien quelles qu'elles soient, y compris dans leurs formes banales et routinières.

\subsubsection{Le voisinage}

19 Le positionnement du logement avec ses abords immédiats et les relations qu'entretient le ménage avec le voisinage s'inscrivent dans la lignée de l'observation des pratiques habitantes. Il s'agit pour le chercheur de questionner la nature des liens 
qui existe entre les habitants et leur entourage, qu'ils soient affectifs, interdépendants, contraints, choisis, recherchés, etc., et l'importance que revêt le réseau relationnel à leurs yeux. En observant l'espace du chez-soi dans sa configuration matérielle, le chercheur est appelé à s'intéresser par extension aux questions de frontières et de définitions de l'espace privé, aux formes de repli ainsi qu'à leurs logiques sécuritaires. L'idée de partition nette entre l'espace privé et public dans un souci aussi gestionnaire que sécuritaire pose ainsi directement la question des formes de territorialisation de l'individu.

\subsubsection{La dispersion du chez-soi dans l'espace, la domus éclatée}

20 La compréhension des logiques résidentielles conduit à élargir l'analyse aux autres lieux de résidences fréquentés par l'habitant et aux relations qui existent entre eux. La multi-résidence fait appel à l'expérience spatiale de chacun, soit l'histoire résidentielle personnelle et le capital culturel migratoire accumulé. En contexte tanzanien, habiter plusieurs lieux à la fois est une pratique courante qui concerne toutes les catégories sociales, en raison de l'exode rural et de la décohabitation des générations qui l'accompagne. La répartition inégale à l'échelle nationale des secteurs d'activité comme le tourisme, l'industrie ou l'éducation rend l'obligation de la mobilité professionnelle plus nécessaire encore et contribue à ce que les individus se délocalisent et multiplient leurs lieux de résidence. La circulation entre ces différentes résidences et le maintien des liens avec la région d'origine (par la construction d'une propriété "au pays » comme il est fréquent d'observer) participent à l'entretien d'une forme d'archipel résidentiel. Alors qu'elle désigne une aire de circulation géographique globale, la multirésidence constitue - dans la même veine que le décor, les activités domestiques et le voisinage proche - un patrimoine tout aussi observable pour le chercheur. Elle peut être présente au travers des discours, des objets et de certaines pratiques qui rappellent constamment l'éclatement de la domus et le souci de mettre en réseau ses différents points d'ancrage.

21 Pour conclure, si la maison est capable d'abriter la dialectique mouvement/ sédentarisation et de produire des identités métisses, c'est qu'elle garde aujourd'hui, en temps de changement d'échelle, un caractère profondément dynamique et moderne. La «domus » correspond d'autre part à l'objectif ici recherché de rendre compte à la fois des réalités pragmatiques du quotidien observé et des points de vue personnels objectivés sur l'espace du «chez ». Dans une même optique, la lecture de l'image habitée sous ses différentes formes pratiques et domestiques ainsi que dans sa mise en réseau avec l'extérieur rappelle combien l'attachement matériel est important pour l'individu qui personnalise son territoire. Nous trouvons dans le contexte d'Arusha et ses alentours autant d'illustrations possibles de ces chez-soi vécus au quotidien en espérant apporter le complément ethnographique à une démarche anthropologique encore en chantier.

\section{Le chez-soi dans ses formes abstraites}

«(...) de fait dans la logique du vécu, le rapport spatial ne saurait se borner aux seuls espaces des pratiques quotidiennes, épisodiques ou exceptionnelles. L'espace des «métastructures individuelles » recèle aussi la part du rêve et des images, celle des symboles et des représentations ${ }^{4}$. » 
Après avoir insisté sur le rôle "silencieux " mais visible de l'habitation en dur, sur l'organisation pragmatique qui la distingue et contribue à fournir au chercheur son matériau ethnographique, plaçons-nous désormais du côté de l'habitant. Nous nous intéressons aux citoyens ordinaires et aux cheminements au fil desquels chacun « invente » le territoire en fonction de ses propres modèles. Nous partons de l'approche anthropologique selon laquelle le territoire est construit et représente un mode de pensée mais aussi d'action qui prend sens dans un contexte. Nous souhaitons d'abord mettre en évidence ce qui conduit l'acteur à s'auto-identifier aux territoires du chezsoi. Nous choisissons pour cela l'exemple déterminant de la mobilité spatiale qui amène à multiplier les expériences résidentielles dans un processus de familiarisation avec le territoire. D'autres enjeux tels que la dimension affective et les questions d'appellation et de délimitation du chez-soi seront par la suite évoqués. Nous nous arrêterons en second temps sur les représentations de l'espace au quotidien, plus précisément sur les expressions géographiques et symboliques collectives de la territorialité vécue, en réunissant sur le terrain le point de vue des habitants sur leur environnement urbain, leur place, leur citoyenneté abstraite, leur conscience diasporique et cosmopolite. Enfin un dernier volet sera l'occasion de nous pencher sur les images de chez-soi faisant l'objet d'un rappel via le rêve et l'imaginaire. Toujours à la recherche des stratégies d'autonomie individuelles ou collectives autour du chez-soi, nous nous arrêterons sur le rêve de maison qui fait appel aux dimensions intimes que sont le souvenir et les liens entretenus avec le premier chez-soi et le projet de construction visant à la réalisation de soi.

\subsection{Chez-soi : de quel territoire s'agit-il ?}

Le propos n'est pas de faire la typologie des définitions du territoire en sciences sociales. La question de la terminologie reste néanmoins essentielle car elle doit rendre compte d'une réalité globale complexe. Nous insistons de nouveau sur l'importance de reconsidérer l'épaisseur du quotidien comme support et cadre de la réflexion menée sur le chez-soi avec les termes d'espaces de vie, d'espace vécu, pratiqué, imaginé, d'espace social et de territoire du quotidien dont on peut déplorer aujourd'hui une forme de banalisation. Le territoire ici convoité est construit par l'acteur. Il s'agit d'un compromis entre son vécu quotidien et le produit de son imaginaire. Chacun trace ses itinéraires quotidiens et cultive ses routines en fonction de son rapport aux lieux, du souvenir profonds de ses racines, de son expérience résidentielle. Chacun encore dispose d'une palette plus ou moins large (choix, contraintes) de pratiques qui marquent profondément ces rapports aux lieux, ces racines, ces nouveaux aménagements.

\subsubsection{Mobilité et chez-soi}

C'est par la mobilité et les diverses expériences résidentielles qui en découlent que l'individu se territorialise et s'achemine vers l'idée de chez-soi. Qu'elle soit ponctuelle, quotidienne, exceptionnelle ou routinière, la mobilité (considérée ici dans sa définition anthropologique), dépasse la réalité géographique pour se mesurer en termes d'expériences accumulées du "proche » et du "lointain ». En ce qu'elle représente la capacité à substituer un lieu à un autre et le rattachement à celui-ci, l'ancrage, la 
mobilité aboutit donc à une dissociation spatiale entre plusieurs milieux de vie et forme concrètement le noyau dur qui structure l'expérience de vie quotidienne.

C'est sans doute à partir de là que l'individu construit ses propres repérages, ses espaces personnels, de connaissance, le sentiment d'être et de se sentir de quelque part. Le chez-soi dépasse en outre le seul sentiment d'appartenance à l'espace résidentiel. Caractérisé par une impression de familiarité, un rapport personnel et socialisé entre l'individu et l'espace, il dessinerait une "aire émotive " plus globale nourrie par le sentiment d'appropriation qu'éprouve l'acteur à l'égard d'un espace qui lui appartient autant qu'il s'y sent lui appartenir. Le processus identificatoire (chez) et identitaire (soi) qu'entreprend l'individu vis-à-vis du territoire ne se fait pas sans difficultés. Celle de se reconnaître dans un territoire sans savoir ni même vouloir le délimiter par exemple ; accepter de se reconnaître dedans alors qu'il nous dépossède en même temps d'une part de nous-même ; être et se sentir par obligation de quelque part pour agir et être reconnu au niveau politique.

\subsubsection{Définir et nommer le territoire}

Le problème de savoir quel contenu donner au territoire et plus précisément au territoire du chez-soi conserve donc son lot de subtilités. «Un territoire c'est d'abord une convivialité, c'est du concret et de l'abstrait, un savant mélange de matériel et d'idéel " ", écrivait le géographe Joël Bonnemaison. Le sentiment d'appartenir au territoire est d'autant plus subtil qu'il est souvent inconscient (cela va de soi). Nous retrouvons cette flexibilité dans le langage parlé. En kiswahili en contexte tanzanien, par exemple, l'idée de «chez soi " a recours à plusieurs traductions et associations d'idées possibles. Elle est souvent spontanément désignée en fonction des liens qui unissent ou séparent l'individu d'avec la famille et le groupe, comme avec kwetu (qui désigne «chez les nôtres » ou les lieux d'origine du clan) et nyumbani (qui correspond davantage au chezsoi reconstruit du ménage nucléaire). Puis le rajout d'attributs ou d'adverbes jugeant du caractère physique, topographique des lieux comme avec kijijini (au village), mjini (en ville), pwani (sur la côte), milimani (en montagne), pale (ici ou là-bas proche), kule (làbas lointain). poursuit l'effort de retranscription linguistique des réalités du chez-soi.

Au-delà de l'impression de sécurité réelle et affective que génère le territoire et qui rapproche les habitants d'un même lieu, examinons à partir de l'expérience urbaine comment le chez-soi repose sur les menus actes de la vie banale qui façonnent des ambiances particulières ou des images collectives comme des représentations mentales communes.

\subsection{Expressions géographiques et symboliques de la territorialité vécue}

Après avoir rappelé les nombreux facteurs qui entrent en jeu dans la constitution de l'espace du chez-soi et insisté sur le rôle de la mobilité dans l'identification au territoire, arrêtons-nous sur le moment présent, soit la territorialité vécue telle qu'elle est représentée et discutée par nos interlocuteurs. Nous choisissons d'observer les modes de vie de chacun au travers de l'apprentissage et des pratiques de la ville qu'en font ses habitants. L'ouverture à la diversité et à la socialisation qu'offre le milieu urbain encourage l'individu à se positionner dans la société contemporaine. Comment 
l'acteur construit-il ses propres visions du monde, et a posteriori celles du chez-soi, à partir de l'expérience de l'urbanité?

Nous réhabiliterons dans un second temps les différents points de vue des habitants en examinant certaines de leurs représentations urbaines et en éclairant certains traits qui caractérisent à leurs yeux le mieux l'endroit. Nous verrons par ailleurs ce que la ville et l'environnement social global apportent à l'individu en termes de jugements et de revendications. Tout en resituant quelques aspects de notre démarche, nous voulons réhabiliter le social à travers les individus et apporter une visibilité sur leur territorialité au quotidien. Lisibilité encore trop absente des travaux de recherches contemporains en sciences sociales.

\subsubsection{Pratiquer la ville - l'exemple d'Arusha}

Il s'agit de rappeler en premier lieu la dynamique des espaces. Il est nécessaire pour cela de remettre en cause le mythe de la sédentarité qui consacre une coupure entre les localités urbaines et rurales longtemps présentées comme deux territoires mutuellement exclusifs. On constate dans la Tanzanie d'aujourd'hui à quel point la ville et la campagne sont reliées (par les différents réseaux sociaux et d'intercommunications). À Arusha, par exemple, la figure du Massaï qui circule en permanence et de manière rotative entre ces deux milieux le confirme notamment. Toujours soucieux de nous raccrocher au réel, nous questionnons d'abord l'acteur sur ses activités urbaines pour tenter de comprendre quels sont ses itinéraires, trajectoires, réseaux, repérages. Nous observons à ce titre comment s'opèrent l'accès à la ville et la fréquentation de celle-ci (quels sont les moyens de circulation utilisés, quelle distance sépare les lieux d'habitation des repères urbains donnés, etc.). Dans les villes tanzaniennes moyennes comme Arusha, la familiarisation avec les espaces urbains s'effectue d'autant plus souvent par la pratique physique de celle-ci (circulation à pieds). Même si chacun vit ses interactions et apprentissages avec la ville en fonction de ses origines socio-spatiales, ses ressources et ses capacités, il nous parait important de continuer à solliciter chez l'acteur une pensée rétrospective, critique et distanciée de l'environnement au sein duquel il évolue.

Dans le discours de nos habitants, la ville d'Arusha est pour beaucoup associée aux obligations professionnelles. En semaine, l'individu s'y rend ou y passe tôt le matin (notamment par la gare routière) avant de rejoindre les lieux de son travail, puis s'en échappe de la même manière en fin de journée pour regagner son habitation. Pour l'acteur, la fréquentation de celle-ci se limite à une routine quotidienne conditionnée par son propre transport d'un endroit à un autre. Pendant le week-end, Arusha devient le théâtre d'activités ludiques et à caractère cérémoniel, avec les sorties au marché alimentaire central ou au marché aux fripes (mitumba), les courses à l'hypermarché Shoprite, la fréquentation des différentes églises, mosquées, temples ou les sorties familiales et endimanchées dans les quelques hôtels de la ville ou de ses alentours. Des convois festifs et à caractère ostentatoires organisés lors de mariages ou d'autres cérémonies paradent dès le vendredi soir à plusieurs endroits stratégiques de la ville, complétés par des séances rituelles de photographies organisées aux différents rondspoints de la ville (Impala, Mwenge, Clock-Tower). D'autres activités ludiques comme les sorties dans les hôtels de luxe, boîtes de nuit ou cafés-restaurants fréquentés par les touristes, où il est possible d'y regarder la télévision, de jouer au billard, de consommer de l'alcool ou de danser sont pratiquées par certains de nos interlocuteurs. Enfin pour 
les habitants majoritairement musulmans du quartier central du marché, la consommation de grillades ou du café préparés directement dans la rue à la manière des habitants de la côte représente un autre exemple de sorties familiales de fin de semaine.

\subsubsection{Propriétés de la ville d'Arusha} contribué à transformer leurs représentations des lieux et à leur donner une identité forte. Malgré une large visibilité dans l'espace public urbain les monuments bâtiments historiques de la ville, comme l'ancien fort allemand (le Boma) aujourd'hui reconverti en musée d'histoire naturelle, le musée de la déclaration d'Arusha ainsi que l'ensemble du patrimoine architectural (maisons de style colonial, indien ou moderniste) sont en général peu évoqués. Les écoles religieuses, construites au début $\mathrm{du} \mathrm{xx}^{\mathrm{e}}$ siècle par les missionnaires sur les collines qui surplombent la ville d'Arusha (écoles secondaires d'Ilboru et d'Enaboishu par exemple), où furent formées plusieurs élites actuelles du pays, le sont davantage, tout comme le bâtiment des Nations Unies du centre-ville, longtemps demeuré spectaculaire à l'échelle d'Arusha. Nous 
remarquons en revanche un entrain collectif exprimé à l'égard des nombreuses constructions en cours. Celles-ci paraissent nourrir à tous les échelons de la société des fantasmes de modernisation au détriment du patrimoine historique bâti qui, alors qu'il est régulièrement entretenu par les autorités, semble pour beaucoup totalement insignifiant. Sans entrer dans les détails, rappelons également à quel point la ville d'Arusha bénéficie, de par sa proximité avec les parcs nationaux et les territoires circulatoires massaïs environnants, d'une identité particulière basée sur la gestion de la nature et le profit qu'elle en tire. Le dynamisme économique, lié en majorité au secteur du tourisme, est sans doute le critère le plus représentatif de la ville aux yeux de ses habitants. Enfin la fertilité, le climat d'altitude et la proximité avec les deux plus grandes montagnes de la région (le mont Meru et le mont Kilimanjaro) sont autant de représentations identitaires et symboliques qui participent à la définition de la ville.

On peut en conclusion aborder la ville d'un point de vue socio-affectif en la considérant comme un milieu apte à engendrer un type de sociabilité. La ville est une dominante qui crée une ambiance spécifique. Il y a les espaces comme le quartier de résidence où il faut habiter pour se sentir chez soi, le centre urbain comme territoire commun, puis d'autres espaces dits neutres où l'on est ni chez l'un ni chez l'autre. Réfléchir sur la territorialité, c'est chercher à identifier la place de l'espace dans les processus de socialisation. En interrogeant le caractère plus ou moins localisant des imaginaires, nous cherchons à questionner l'habitant sur sa définition d'une pensée égocentrique ou socio-centrique, représentative, locale, autochtone... Le milieu urbain propose en effet à l'individu une ouverture sur le monde en réunissant l'apprentissage de l'espace, de ses pratiques et d'autrui. Ce positionnement de l'individu dans la sphère sociale quotidienne est déterminant pour notre réflexion, il semble en effet nécessaire à l'émergence d'un désir de sédentarisation et à la production d'un chez-soi. En écho à ces dynamiques d'ouverture sur le monde et la société, revenons maintenant à des domaines plus personnels.

\subsection{Le chez-soi rêvé}

37 Nous voudrions terminer la synthèse de nos idées motrices en accordant une place à l'imagination de l'acteur dans un mouvement de retour sur soi. Le rêve, en ce qu'il offre à l'individu un refuge personnel, constitue le territoire des possibles, des fantasmes et des ambitions. Poursuivant l'interrogation de l'acteur en quête d'une identité spatiale, de sens, nous nous intéressons désormais à celui qui choisit d'autres espaces à partir de ses propres ressources. Examinons ici schématiquement ce que le rêve de maison et sa concrétisation, à travers la construction et l'accession à la propriété, revêtent pour l'individu, au regard de sa propre trajectoire et ascension personnelle comme dans le maintien des liens avec le pays natal.

\subsubsection{Le rêve de maison}

Nous l'avons vu plus haut, la maison correspond à un enracinement matériel et symbolique. Nous nous intéressons ici au projet dans sa totalité, depuis sa genèse jusqu'à sa réalisation. Nous questionnons d'emblée l'intérêt et les dynamiques personnelles qui ont conduit l'individu sur la voie de la propriété. Le processus de construction de la maison sur la terre natale nous intéresse tout particulièrement car il fait intervenir le maintien des liens (et leur transversalité) qui existe entre la région de 
vie actuelle et le chez-soi natal. En Tanzanie, la maison est le lieu idéal de transfert des économies. Face à l'incompétence des pouvoirs publics, elle s'apparente en quelque sorte à un système d'assurance qui vise à augmenter les ressources. Le projet de maison représente donc en premier lieu un objectif lourd de sens pour l'individu comme le défendent les sociologues du mouvement :

«les nouveaux modes de spatialisation de la vie sociale supposent que le projet individuel est devenu à la fois une condition d'efficacité collective et de réalisation de soi. Le contrôle social passe volontiers à travers la manière de rémunérer le projet individuel. À l'inverse l'absence de projet individuel ou l'incapacité de le concrétiser induit facilement une séquence d'exclusion ${ }^{6}$ ».

Dans la suite de notre ethnographie, nous questionnons l'attachement à la terre, en examinant comment celle-ci fut transmise, si le terrain provient du patrimoine familial ou non. Puis nous interrogeons le propriétaire sur les modèles choisis pour la construction (influences, matériaux, etc.). Une fois construite, nous étudions la fréquentation de la maison ainsi que la corrélation entre les visites et les "grandes » occasions (retraite, vacances, cérémonies, etc.). Encore une fois, il s'agit de poser la question de la multifonctionnalité de la maison (la maison répond-elle à des fonctions polyvalentes ou d'accueil ?). Nous désirons comprendre quel est le lien qui unit les propriétaires aux nouveaux lieux (qui occupe les lieux en leur absence, la maison estelle confiée, louée, comment assurent-ils sa rentabilité ?).

En ce qu'elle cristallise un projet d'amélioration du niveau de vie et d'ascension professionnelle, la maison fait partie d'une stratégie individuelle d'ascension. Il en est de même pour l'émigration qui vise à augmenter ses propres manœuvres. Si les mouvements résidentiels sont nombreux en Tanzanie, ils ne sont bien évidemment pas toujours choisis. De même, si le projet de construire au pays natal est dans la plupart des cas profondément souhaité, il ne se concrétise pas toujours en ces termes. À Arusha par exemple, beaucoup d'habitants originaires d'autres régions de Tanzanie cherchent à y acquérir un terrain plutôt que sur leur terre natale, sans doute motivés par les facteurs socio-économiques qui caractérisent les lieux.

41 Les différentes étapes du projet ont toutes leur intérêt anthropologique. Il y a d'abord le paradoxe de la maison qui inaugure l'enracinement familial par cette première possession mais aussi disperse les membres de la famille du fait de l'émigration; la question des reconfigurations familiales et statutaires qu'elle inaugure (le couple qui construit sa maison devient une unité économique nouvelle, les repositionnements intergénérationnels, le rôle de chacun, la répartition des rôles dans le projet, etc.) ; puis les éventuels problèmes de cohabitations.

À travers ce cheminement, l'objectif est donc de réhabiliter l'acteur/habitant, ses propres souhaits et ce qu'il pense de ses choix. Nous questionnons ce dernier sur ses différentes formes "d'utopie résidentielle ». Il s'agit notamment du désir d'accéder au statut de propriétaire et ce que cela représente aujourd'hui au regard de la société tanzanienne en mouvement, de sa volonté de reproduire une maison qui lui rappelle celle où il est né, de son envie d'affirmer son changement de statut et sa nouvelle urbanité, mais aussi de l'affect qui y est ajouté, où l'habitat peut être survalorisé pour exalter le passé afin de fixer son identité. D’après Philippe Bonnin, «il peut en effet résulter une attitude ambivalente entre, d'un côté, le sentiment d'appartenance au pays d'origine et aux ascendants a ce qu'on y a laissé et, de l'autre, le sentiment de promotion, de réalisation de soi et l'autonomisation de la culture d'accueil ${ }^{7}$ ». Le retour sur soi a donc 
permis d'évoquer l'importance que revêtent les points d'attache (motivations), les points d'ancrage (repères identitaires) et ceux de renouvellement dans l'analyse du chez-soi. Revenir sur l'acteur laisse transparaître les stratégies et les conduites d'autonomie qui le guident, mais aussi sa propre créativité en laissant le débat ouvert sur le potentiel des migrants à déconstruire les modèles et à créer des chez-eux originaux en multipliant les jeux de recomposition d'éléments empruntés à différents répertoires (rural/urbain, artisanal/industriel, traditionnel/moderne, local/étranger, savant/populaire...).

\section{Conclusion générale}

Notre réflexion s'inscrit dans le cadre d'une socio-anthropologie de l'habitat qui prend en compte les dimensions pratiques de la vie quotidienne. Nous nous intéressons à l'habitant, l'habitat et ce qu'il produit comme formes d'habiter, de cohabitations et de co-présences. Notre approche privilégie un rapport souple au territoire. Elle évolue dans un ensemble de jeux visibles et invisibles, dans un espace composé d'une géométrie variable qui ne circonscrit pas des aires délimitées et que nous avons choisi d'appeler dès le début de nos recherches «chez-soi ».

Dans la présente synthèse de nos idées phares, nous avons privilégié l'argumentation du champ de notre problématique à la restitution détaillée de nos données ethnographiques. Il nous a semblé important d'expliquer point par point ce qui, selon nous, «façonne » le chez-soi, dans une démarche constructive et exploratoire. Il a fallu pour cela réhabiliter les éléments recontextualisants suivants : d'abord l'acteur et sa propre restitution en tant que sujet pensant de la société, son espace de vie quotidienne visible et fonctionnel, ses différentes territorialisations dans l'espace social, puis ses propres représentations et productions de l'espace. Nous avons néanmoins pris soin de laisser transparaître à travers le plan théorique de notre réflexion le déroulement de nos enquêtes et la nature de notre matériau ethnographique.

Le rapport à la modernité et aux dynamiques sociétales contemporaines a par ailleurs été évoqué à travers la dialectique de l'habiter par la ville et de la ville par l'habiter. En examinant ce que le chez soi dit sur l'ici, l'ailleurs et sur soi, l'objectif est de rechercher ce qui constitue pour l'individu dans chacun de ces foyers (domestique et urbain) des sources d'inspiration et de renouvellement personnel. C'est à partir de là que nous souhaitons acheminer cette réflexion vers l'autonomie des acteurs et les conditions de réalisation de soi telles qu'elles sont aujourd'hui possibles dans la société tanzanienne en mouvement. Le projet de construire sa propre maison, le rêve de celle-ci, ou les réminiscences du premier chez-soi sont autant d'exemples de ce temps pour soi que nous aimerions par la suite approfondir.

Ce travail révèle d'autres champs d'observation qui sont en cours de problématisation. Nous questionnons par exemple la pertinence d'une analyse portant sur la dialectique autochtones/néos et leurs éventuels conflits; sur la mutation du collectif vers l'individuel; sur la combinaison possible entre des formes d'individualisation et des solidarités nouvelles, etc. Enfin, après avoir rappelé l'existence de lacunes concernant les domaines du quotidien dans les sciences sociales appliquées à des contextes africains, nous restons préoccupés par l'idée de trouver un compromis méthodique concernant l'ethnographie et sa retranscription. Nous cherchons en effet à nous situer 
entre une socio-anthropologie qui serait pratique (par l'examen des formes d'habiter), mais aussi psychologique, par le récit de vie et l'approche biographique.

Nos principaux défis consistent donc à mêler les apports de plusieurs méthodes d'enquêtes. La méthode biographique d'abord, qui permet de reconnaître au savoir de l'individu une valeur sociologique, en révélant ce qu'il y a d'actif et de passif en lui, sans pour autant empêcher l'homme ordinaire de toute capacité d'initiative ou de conscience critique. Nous nous situons alors en opposition avec les théories comme le fonctionnalisme et le structuralisme qui aboutissent à vider l'ordre social de toute contradiction profonde, à le penser comme un organisme, un système, une structure. L'approche biographique nous intéresse également en ce qu'elle permet de saisir «l'épaisseur du social » dans sa diversité et dans ses contradictions multiples, mais aussi en ce qu'elle met en évidence les mécanismes intermédiaires du rapport entre l'individuel et le social. Le récit de vie ensuite, qui produit un matériau susceptible d'exprimer à la fois le poids des déterminations sociales dans les trajectoires individuelles et le rapport des acteurs à ces déterminations (et donc à partir de là leur créativité propre). Cette seconde approche nous intéresse en outre en ce qu'elle tente de saisir le rapport dialectique entre l'individu produit de l'histoire et l'individu producteur d'histoire.

La poursuite de ces recherches devra donc continuer de saisir les subjectivités de l'expérience vécue afin d'apporter une lecture nouvelle des formes de chez-soi visibles et possibles aujourd'hui en Tanzanie.

\section{BIBLIOGRAPHIE}

Bonnemaison, J. (1979). « Voyage autour du territoire. » L'Espace géographique, $\mathrm{n}^{\circ} 4$.

Berthelot, J.M., Hirschhorn, M. (1996). Mobilités et ancrages. Vers un nouveau mode de spatialisation? Paris : L'Harmattan.

Bonnin, P., de Villanova R. (1996). D'une maison l'autre. Parcours et mobilités résidentielles. Paris : Créaphis.

de Villanova, R., Leite, C., Raposo, I., (1994). Maisons de rêve au Portugal, éd. Paris : Créaphis.

Digneffe F., Beckers, M. (1995). « De l'individuel au social : l'approche biographique. » In L. Albarello, F. Digneffe et al, Pratiques et méthodes de recherche en sciences sociales. Paris : Armand Colin.

di Méo, G. (1996). Les Territoires du Quotidien. Paris : l'Harmattan.

Spear, T. (1997). Mountain Farmers. Moral economies of land and agricultural development in Arusha and Meru. Oxford : James Currey.

Tarrius, A. (2000). Les nouveaux cosmopolitismes. Mobilités, identités, territoires. Paris : Éditions de l'Aube. 


\section{NOTES}

1. P. Tizon, « Qu'est-ce que le Territoire?» in Di Méo, G. (1996), p. 25.

2. Tarrius (2000), p. 126.

3. Bonnin \& de Villanova (1996), p. 10.

4. G. Di Méo, «Territoire vécu et contradictions sociales : le cas de la vallée d'Aspe », in Di Méo (1996), p. 69.

5. Bonnemaison (1979) : 255 et 261.

6. Berthelot \& Hirschhorn (1996).

7. Bonnin \& de Villanova (1996) : 12.

\section{INDEX}

Index géographique : Tanzania| Tanzanie

\section{AUTEUR}

\section{MATHILDE DE BLIGNIÈRES}

Doctorante en anthropologie sociale à l'École des Hautes Études en Sciences Sociales( EHESS) à Paris, et boursière de l'IFRA. 